\title{
Numerical simulation of bubble detachment at submerged orifice and analysis of interface stability *
}

\author{
Xian-xian Yu (于涃涃 $)^{2}$, Yi-wei Wang (王一伟) ${ }^{1,3}$, Chen-guang Huang (黄晨光) $)^{1,3}$, Te-zhuan Du (杜特专) $)^{1,3}$ \\ 1. Key Laboratory for Mechanics in Fluid Solid Coupling Systems, Institute of Mechanics, Chinese Academy of \\ Sciences, Beijing 100190, China \\ 2. Beijing Institute of Mechanical and Electrical Engineering, Beijing 100074, China \\ 3. School of Engineering Sciences, University of Chinese Academy of Science, Beijing 100049, China
}

(Received April 12, 2016, Revised July 20, 2016, Accepted October 9, 2016, Published online December 29, 2018)

(C) China Ship Scientific Research Center 2018

\begin{abstract}
This paper presents the experimental and numerical results of the bubble detachment from a submerged orifice at a constant gas flow rate. The compressible large eddy simulation combined with the volume of fluid method is adopted in the simulation and is validated by experiment. The transition criterion from the elongation stage to the detachment is obtained. In the detaching stage in the simulation, the distributions of the pressure and the surface tension on the cylindrical bubble neck are obtained. The Rayleigh-Plesset equation in the cylindrical coordinate frame is used to describe this process. Based on the comparison between the numerical results and the equation analysis, a reference value of the uncertain integral parameter in the equation is determined.
\end{abstract}

Key words: Bubble interfacial dynamics, large eddy simulation, instability criterion, Rayleigh-Plesset equation

\section{Introduction}

The process of the bubble formation at the submerged orifice is very important in many engineering applications, such as the bubble column, the gas-liquid separator, and the cavitation. Owing to its practical importance, the phenomenon of the bubble formation and the pitch-off was widely studied theoretically and experimentally for decades.

A large number of investigations of the bubble formation at a submerged orifice were reviewed in Zhang et al. ${ }^{[1]}$, Legendre ${ }^{[2,3]}$, Gerlach et al. ${ }^{[4-6]}$ and Kulkarni et al. ${ }^{[7]}$. At a low gas flow rate, the formation of the bubble is dominantly affected by the buoyancy and the surface tension, described by the Young-Laplace equation ${ }^{[4]}$. Gerlach et al. ${ }^{[4-6]}$ did a comprehensive study of the bubble formation at a submerged orifice at low and medium constant gas

* Project supported by the National Natural Science Foundation of China (Grant No. 11772340, 11672315, the Youth Innovation Promotion Association CAS(Grant No. 2015015).

Biography:Xian-xian Yu (1987-), Female, Ph. D.,

E-mail:yuxianxian1987@163.com

Corresponding author: Yi-wei Wang,

E-mail:wangyw@imech.ac.cn flow rates. They proposed a theoretical model of the quasi-static bubble formation at very small flow-rates. It was observed that the strong vortex generated by a large leading bubble influences the growth of the subsequent bubble and turns the period-1 bubbling regime into the period-2 bubbling regime. Jamialahmadi et al. ${ }^{[8]}$ found that the bubble volume increases with the increase of the orifice radius. The frequency of the bubble formation increases with the increase of the gas flow rate. At a high gas flow rate, the bubble size increases significantly as the liquid viscosity is increased. According to Zhang's work ${ }^{[1]}$, the bubbling phenomena above a submerged orifice can be divided into four regimes, the single bubbling, the pairing, the double coalescence and the triple bubble formation. The experimental results and the theoretical prediction both demonstrate that the bubble interaction is the primary reason for the aperiodic characteristics of the bubble detachment. The gas bubble formation in liquid was extensively studied ${ }^{[9-10]}$. Zhu et al. ${ }^{[11]}$ and Xie et al. ${ }^{[12]}$ studied the dynamic bubbling behaviors in a bubble column with a micro-orifice by experiments. Martin ${ }^{[13]}$ studied the effect of the orifice configuration on the coalescence of growing bubbles. Jian Zhang et al. ${ }^{[14]}$ carried out Visualization experiments and numerical simulations on the bubble dynamic behavior at the submerged 
micron orifice. The outcomes indicate that under a low gas flow rate, the bubble grows and detaches individually, and the bubble formation progress can be differentiated into three stages: nucleation, stable growth, and necking.Karel Vokurka ${ }^{[15]}$ studied wall motion of a bubble freely oscillating in a liquid from the point of view of energy conversions at different instants. It is shown that the time of the bubble scillation can be divided into two distinct intervalslong interval and short interval. Sarhan, A.R. et al. ${ }^{[16]}$ found that the average gas holdup increase with the increase in superficial gas velocity and gas phase density, and decrease with the increase in liquid phase density.

The bubble detachment from a submerged orifice is a complex and key point in the bubble formation process, which draws considerable interest [17-23]. Burton et al. ${ }^{[17]}$ used the cylindrical coordinate version of the general Rayleigh-Plesset equation to describe the bubble dynamics near the pinch region. A 100000 frame-per-second video was used to record the pinch-off of nitrogen gas bubbles in fluids with a wide range of viscosity. They found a power law solution in time, by neglecting the pressure in the bubble. If the external fluid is highly viscous $\left(\eta_{\text {ext }}>100 \mathrm{cP}\right.$ ), the bubble neck radius is proportional to the time before break, $\tau$, and decreases smoothly to zero. If the external fluid has a low viscosity $\left(\eta_{\text {ext }}<10 c P\right.$ ), the radius is proportional to $\tau^{1 / 2}$. Gordillo et al. ${ }^{[18]}$ also revealed that the bubble minimum radius $r_{n}$ decreases in a manner of $\tau \propto r_{n}^{2} \sqrt{-\ln r_{n}^{2}}$ at high Reynolds numbers. In Leppinen's study ${ }^{[19]}$, the axisymmetric pinch-off of an inviscid drop immersed in an ambient inviscid fluid is examined over a range of density ratio $D$. For moderate values of $\mathrm{D}$, the inviscid pinch-off is asymptotically self-similar with both radial and axial length scales, decreasing as $\tau^{2 / 3}$ with velocities increasing as $\tau^{-1 / 3}$, where $\tau$ is the time to the pinch-off. The similar results, independent of the initial conditions for a given value of $D$, can be found in the experiment of Thoroddsen ${ }^{[20]}$. They found that for bubbles in liquid, the radius of the neck reduces in a power law $R \sim \tau^{\alpha}$. The value of $\alpha$ in the power law increases with the increase of the viscosity of the mixture.

Despite many evaluable theoretical and experimental results in literature, so far the detachment mechanism is not clearly explained. In the present paper, we present our numerical and experimental results on the bubble detachment. The transition criterion from the elongation stage to the detachment is obtained and validated. The dynamics of the bubble interface is also analyzed, and the indetermined parameter in the Rayleigh-Plesset equation is confirmed by the comparison of the theoretical and numerical results.

\section{Experimental setup}

The experiment of the bubble formation on a submerged orifice is conducted at the normal pressure and temperature. The experiment setup is sketched in Fig. 1. A glass tank with a stainless steel plate at the bottom is used, as shown in Fig. 2. The cross section of the tank is $500 \mathrm{~mm} \times 500 \mathrm{~mm}$ with an orifice in the middle of the plate. The diameter of the orifice is $2 \mathrm{~mm}$. Compressed air is pumped into the orifice through a long plastic tube and a retaining valve. A flow rate controller is used to control the gas flow rate. In the experiment, the depth of the water in the glass tank is $100 \mathrm{~mm}$ and the gas flow rate is $100 \mathrm{ml} / \mathrm{min}$.

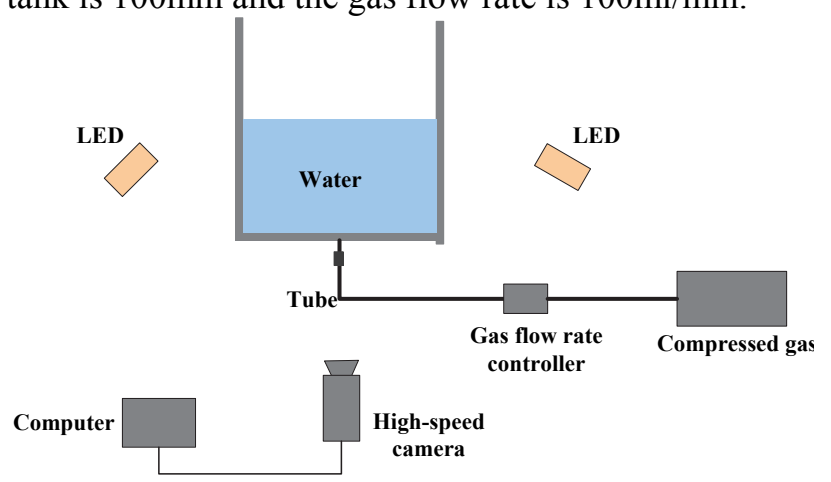

Fig.2 Sketch of experiment setup

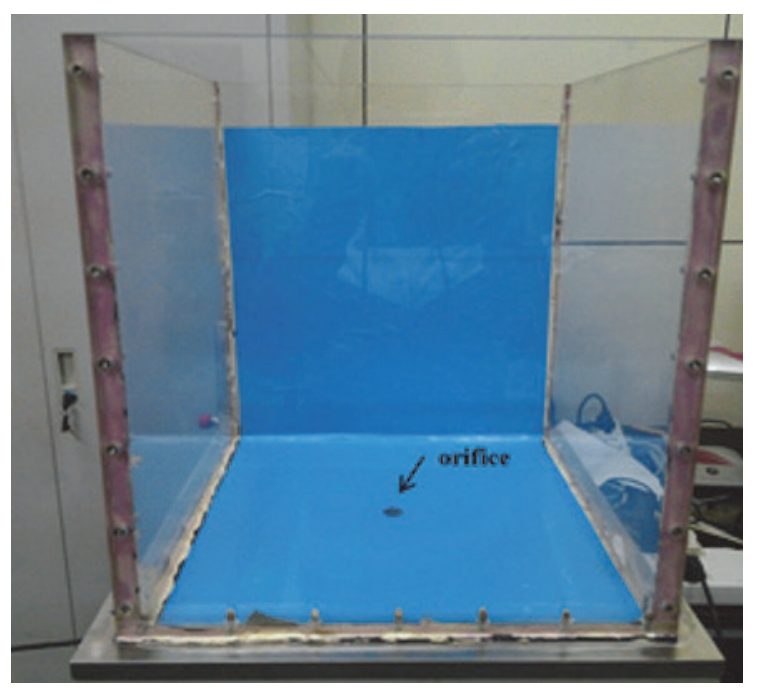

Fig. 4 Glass reservoir 


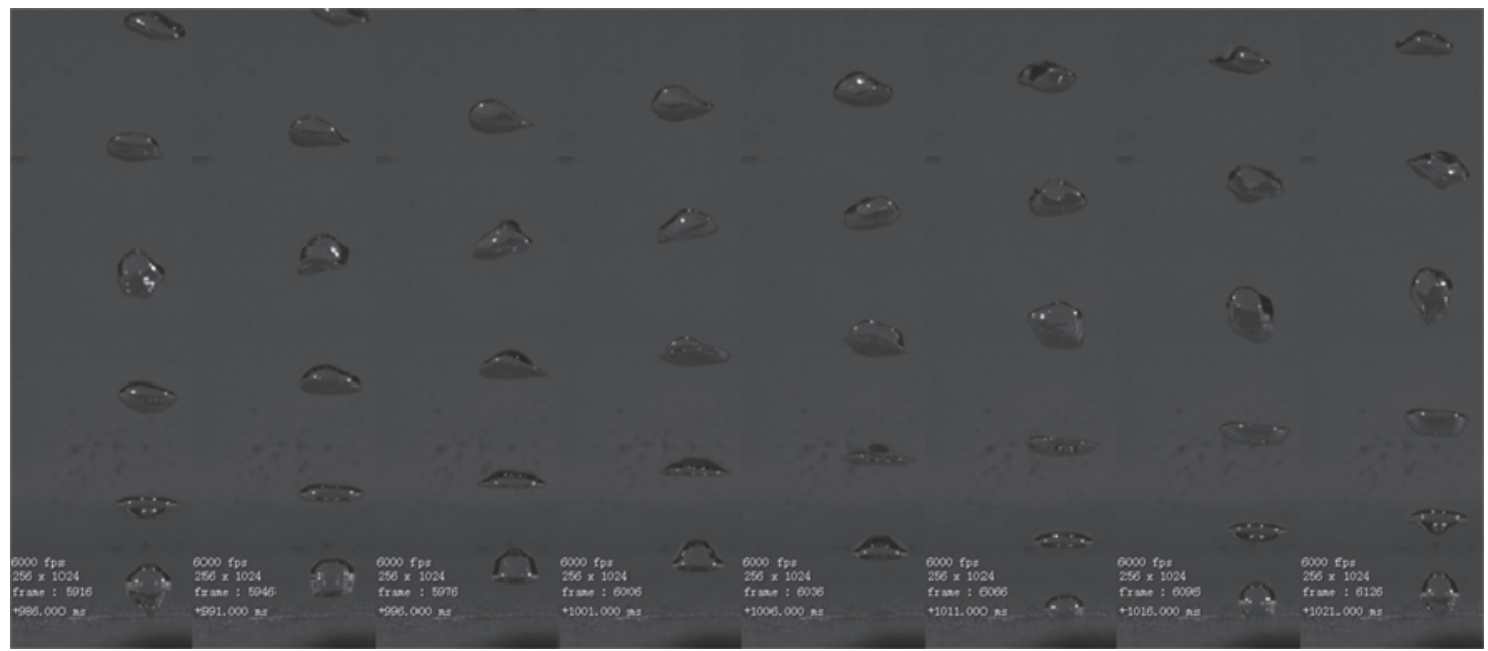

Fig. 5 Bubble pictures at gas flow rate of $100 \mathrm{ml} / \mathrm{s}$ in experiment

\section{Numerical simulation}

The bubble formation is a two phase phenomenon. For the numerical treatment of this problem, the compressible large eddy simulation (LES) combined with a volume of fluid (VOF) method is used in this paper.

\subsection{Governing equations for compressible large eddy} simulation

The Large Eddy Simulation is an intermediate technique between the direct numerical simulation (DNS) of turbulent flows and the solution of the Reynolds-averaged Navier-Stokes equations. For a turbulent flow, the large-scale eddy structures are anisotropic while the smaller ones are supposed to be more isotropic and less affected by the boundary conditions ${ }^{[24]}$. The essential feature of the LES is that the large scales are resolved directly based on the instantaneous Navier-Stokes equation while the small-scales are represented by sub-grid scale models. The governing equations for compressible flows are expressed as:

$\frac{\partial \rho}{\partial t}+\frac{\partial\left(\rho u_{i}\right)}{\partial x_{i}}=0$

$\frac{\partial\left(\rho u_{i}\right)}{\partial t}+\frac{\partial\left(\rho u_{i} u_{j}\right)}{\partial x_{j}}=-\frac{\partial p}{\partial x_{i}}+\frac{\partial \tau_{i j}}{\partial x_{j}}$

$\frac{\partial(\rho E)}{\partial t}+\frac{\partial\left(\rho E u_{i}\right)}{\partial x_{i}}=-\frac{\partial\left(p u_{i}\right)}{\partial x_{i}}+\frac{\partial\left(\tau_{i j} u_{i}\right)}{\partial x_{j}}-\frac{\partial q_{i}}{\partial x_{i}}$

In the above equations, $\rho$ is the flow density, $u_{i}(i=1,2,3)$ are the velocity components and $p$ is the pressure. $\tau_{i j}$ is the shear stress tensor, defined as:

$$
\tau_{i j}=2 \mu S_{i j}-\frac{2}{3} \mu S_{k k} \delta_{i j}
$$

Here, $\mu$ is the dynamic viscosity and $S_{i j}=\frac{1}{2}\left(\frac{\partial u_{i}}{\partial x_{j}}+\frac{\partial u_{j}}{\partial x_{i}}\right)$ is the strain rate tensor.

The total energy per unit mass $E$ is given by

$E=\varepsilon+\frac{1}{2} u_{i} u_{i}$

where $\varepsilon=h-\frac{p}{\rho}$ is the specific internal energy and $h$ is the enthalpy per unit mass. $q_{i}$ is the heat flux vector, obtained as

$q_{i}=-\kappa \frac{\partial T}{\partial x_{i}}$

where $\kappa$ is the thermal conductivity coefficient and $T$ is the temperature.

In the LES, filtering operations are used to separate the large scales from the small ones.

$\bar{f}(x)=\int_{\infty} f(x) \cdot G\left(x-x^{\prime}\right) d x^{\prime}$

where $G$ is the filter function. In the present work, the top-hat filter function is adopted

$$
G\left(x, x^{\prime}\right)= \begin{cases}1 / \bar{\Delta} & \left|x-x^{\prime}\right| \leq \bar{\Delta} / 2 \\ 0 & \left|x-x^{\prime}\right| \leq \bar{\Delta} / 2\end{cases}
$$

where $\Delta=\sqrt[3]{\Delta x \cdot \Delta y \cdot \Delta z}$ is the spatial filter size. 
$\Delta x, \Delta y, \Delta z$ are the sizes of the grid in three directions.

For compressible flows, the Favor-filtering with the filtered variables weighted by the density is used. It is a main difference from the LES for incompressible flows. A Favor filtered variable is defined as

$\tilde{f}=\frac{\overline{\rho f}}{\bar{\rho}}$

The filtered Favor-averaged Navier-Stokes equations are as follows,

$$
\frac{\partial \bar{\rho}}{\partial t}+\frac{\partial\left(\bar{\rho} \tilde{u}_{i}\right)}{\partial x_{i}}=0 p
$$

$$
\begin{array}{r}
\frac{\partial\left(\bar{\rho} \tilde{u}_{i}\right)}{\partial t}+\frac{\partial\left(\bar{\rho} \tilde{u}_{i} \tilde{u}_{j}\right)}{\partial x_{j}}=-\frac{\partial \bar{p}}{\partial x_{i}}+\frac{\partial \tilde{\tau}_{i j}}{\partial x_{j}} \\
+\frac{\partial \tau_{i j}^{S G S}}{\partial x_{j}}+\frac{\partial D_{i j}^{S G S}}{\partial x_{j}} p
\end{array}
$$

$$
\begin{array}{r}
\frac{\partial(\bar{\rho} \tilde{E})}{\partial t}+\frac{\partial\left(\tilde{\rho} \tilde{E} \tilde{u}_{i}\right)}{\partial x_{i}}=-\frac{\partial\left(\bar{p} \tilde{u}_{i}\right)}{\partial x_{i}}+\frac{\partial\left(\tilde{\tau}_{i j} \tilde{u}_{i}\right)}{\partial x_{j}} \\
-\frac{\partial \tilde{q}_{i}}{\partial x_{i}}+\frac{\partial H_{i}^{S G S}}{\partial x_{i}}+\frac{\partial \sigma_{i j}^{S G S}}{\partial x_{j}}-\frac{\partial Q_{i}^{S G S}}{\partial x_{i}} p
\end{array}
$$

The subgrid terms for the required closure in the above equations are as follows,

$$
\begin{aligned}
& \tau_{i j}^{S G S}=\left(\bar{\rho} \tilde{u}_{i} \tilde{u}_{i}-\bar{\rho} \widetilde{u_{i} u_{j}}\right) \\
& D_{i j}^{S G S}=\bar{\tau}_{i j}-\tilde{\tau}_{i j} \\
& H_{i}^{S G S}=\left(\bar{\rho} \tilde{E} \tilde{u}_{i}-\bar{\rho} \widetilde{E u_{i}}\right)+\left(\overline{p u_{i}}-\bar{p} \tilde{u}_{i}\right) \\
& \sigma_{i j}^{S G S}=\overline{\tau_{i j} u} u_{i}-\tilde{\tau}_{i j} \tilde{u}_{i} \\
& Q_{i}^{S G S}=\bar{q}_{i}-\tilde{q}_{i}
\end{aligned}
$$

According to $\operatorname{Lin}^{[25]}, \quad D_{i j}^{S G S}, \sigma_{i j}^{S G S}$ and $Q_{i}^{S G S}$ represent the nonlinearity of the viscous, sub-grid viscous diffusion and sub-grid heat flux. These three terms, which are much smaller than the other terms, are generally neglected. $\tau_{i j}^{S G S}$ and $H_{i}^{S G S}$ are the sub-grid stress and the sub-grid enthalpy flux. These two terms are needed to be modeled.

In the present paper, $\tau_{i j}^{S G S}$ is modeled as $\tau_{i j}^{S G S}=-2 \mu^{S G S}\left(\tilde{S}_{i j}-\frac{\delta_{i j}}{3} \tilde{S}_{k k}\right)+\frac{2}{3} \bar{\rho} k^{S G S} \delta_{i j}$ where the sub-grid eddy viscosity $\mu^{S G S}$ and the sub-grid kinetic energy $k^{S G S}$ are modeled by the kinetic energy one-equation model.

$$
\begin{aligned}
\frac{\partial \bar{\rho} k^{S G S}}{\partial t}+\frac{\partial \bar{\rho} \tilde{u}_{j} k^{S G S}}{\partial x_{j}}=\frac{\partial}{\partial x_{j}} & {\left[\left(\frac{\mu^{S G S}}{\operatorname{Pr}_{t}}+\mu\right) \frac{\partial k^{S G S}}{\partial x_{j}}\right] } \\
+ & P^{S G S}-\varepsilon^{S G S} 00
\end{aligned}
$$

where $\mu^{S G S}=C_{k} \bar{\rho} \Delta \sqrt{k^{s g s}} \quad, \quad P^{s g s}=-\tau_{i j}^{s g s} \frac{\partial \tilde{u}_{i}}{\partial x_{j}} \quad$ and $\varepsilon^{s g s}=C_{e} \frac{\bar{\rho}\left(k^{s g s}\right)^{3 / 2}}{\Delta}$ are the sub-grid kinetic energy production and dissipation, respectively, with $C_{k}=0.094$ and $C_{e}=1.048$.

The sub-grid total enthalpy flux $H_{i}^{S G S}$ is modeled as

$$
H_{j}^{s g s}=-\frac{\mu^{S G S}}{\operatorname{Pr}_{t}} \frac{\partial \tilde{H}}{\partial x_{j}}=-\frac{\mu^{S G S}}{\operatorname{Pr}_{t}}\left(\frac{\partial \tilde{h}}{\partial x_{j}}+\tilde{u}_{i} \frac{\partial \tilde{u}_{i}}{\partial x_{j}}+\frac{\partial k^{s g s}}{\partial x_{j}}\right)
$$

where $\operatorname{Pr}_{t}$ is the sub-grid turbulent Prandtl number and takes the value of 1.0 here.

\subsection{Multiphase Model}

The VOF multiphase model is used to describe the air and water interaction in the present work. $\alpha$ is the volume fraction of the water. The density and the viscosity of the mixture can be expressed as

$$
\begin{aligned}
& \rho=\alpha \rho_{l}+(1-\alpha) \rho_{g} \\
& \mu=\alpha \mu_{l}+(1-\alpha) \mu_{g}
\end{aligned}
$$

where the subscripts $l, g$ represent the water and the gas, respectively.

A transport equation for $\alpha$ needs to be incorporated into the filtered equations.

$$
\frac{\partial \alpha}{\partial t}+\frac{\partial\left(\tilde{u}_{i} \alpha\right)}{\partial x_{i}}=0
$$

\subsection{Computational model}

An axisymmetric solution domain is adopted in this paper, as shown in Fig. 4. Chen and Fan ${ }^{[26]}$ indicated that the bubble formation process is not influenced by the side wall (as shown in Fig. 4 at $r=R$ ) 
boundary condition if the wall is placed at a distance greater than 2 times of the bubble diameter away from the bubble surface. In view of this fact, the diameter of the axisymmetric solution domain $R$ in this paper is $50 \mathrm{~mm}$. The orifice is set with the flow rate boundary $Q=100 \mathrm{ml} / \mathrm{min}$ and with the radius $r_{o}=1 \mathrm{~mm}$. The length of the channel $l$ is $4 \mathrm{~mm}$. A pressure outlet boundary is specified at the top wall at $L=100 \mathrm{~mm}$. The walls in the model are set as no slip walls (zero gradient) with a contact angel $\alpha=60^{\circ}$.

The computational model described in this paper is implemented by using the OpenFOAM libraries.

For the simulations presented in this paper, the time discretization is performed using the first order implicit Euler scheme. A second order Gauss interpolation scheme is adopted for the gradient terms combined with the first upwind order scheme for the divergence terms and the Laplacian terms. The pressure-velocity coupling is handled via a Pimple (Simple- PISO method) procedure, as a unique algorithm in Openfoam. In this method, the flow field at every time step is regarded as in a steady flow condition and is solved with a simple steady-state algorithm. The PISO algorithm is used to complete the time stepping. The time step is set small enough to ensure a maximum Courant number, Co, less than 0.5 everywhere in the computational domain.

\section{Results and discussions}

\subsection{Validation of simulation}

A comparison between the simulation and the experiment shows that the bubble behaviors have a periodic nature with a period of about $40 \mathrm{~ms}$, as shown in Fig. 5. Firstly, the bubble forms at the orifice as a sphere. Then the spherical bubble is detached from the orifice and rises up. During the rise, the bubble shape changes from a sphere to an ellipsoid with oscillations. As shown in Fig. 5, the simulation results are in good agreement with those of the experiment.

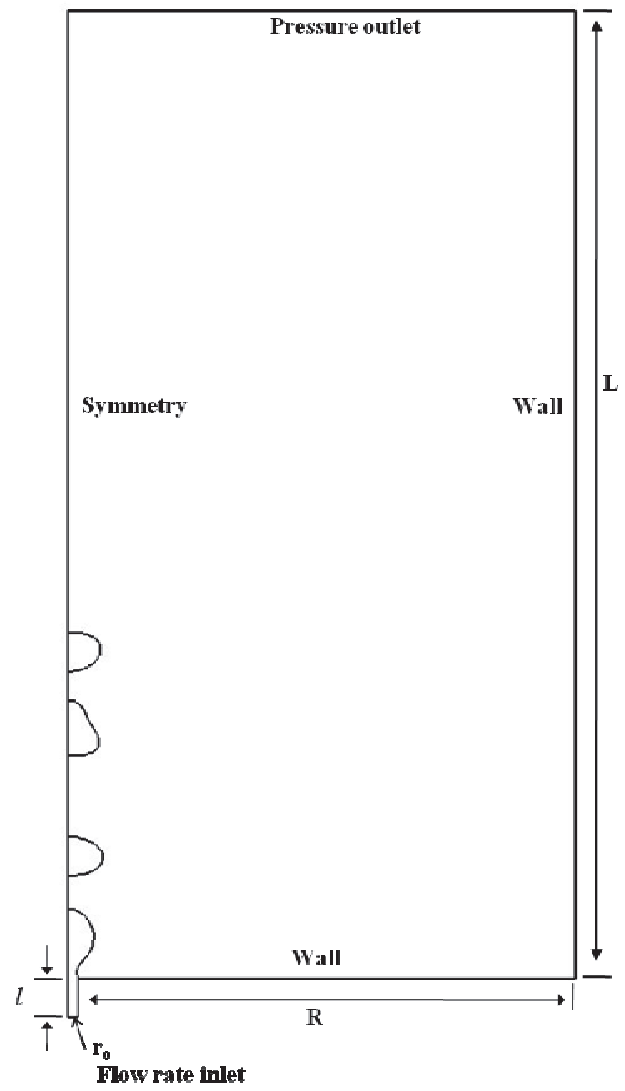

Fig. 6 Simulation domain and boundary conditions

\subsection{Bubble formation}

In the present paper, the bubble formation experiences three stages, as shown in Fig. 7, including the stage I - the bubble expansion, the stage II - the bubble elongation and the stage III- the bubble

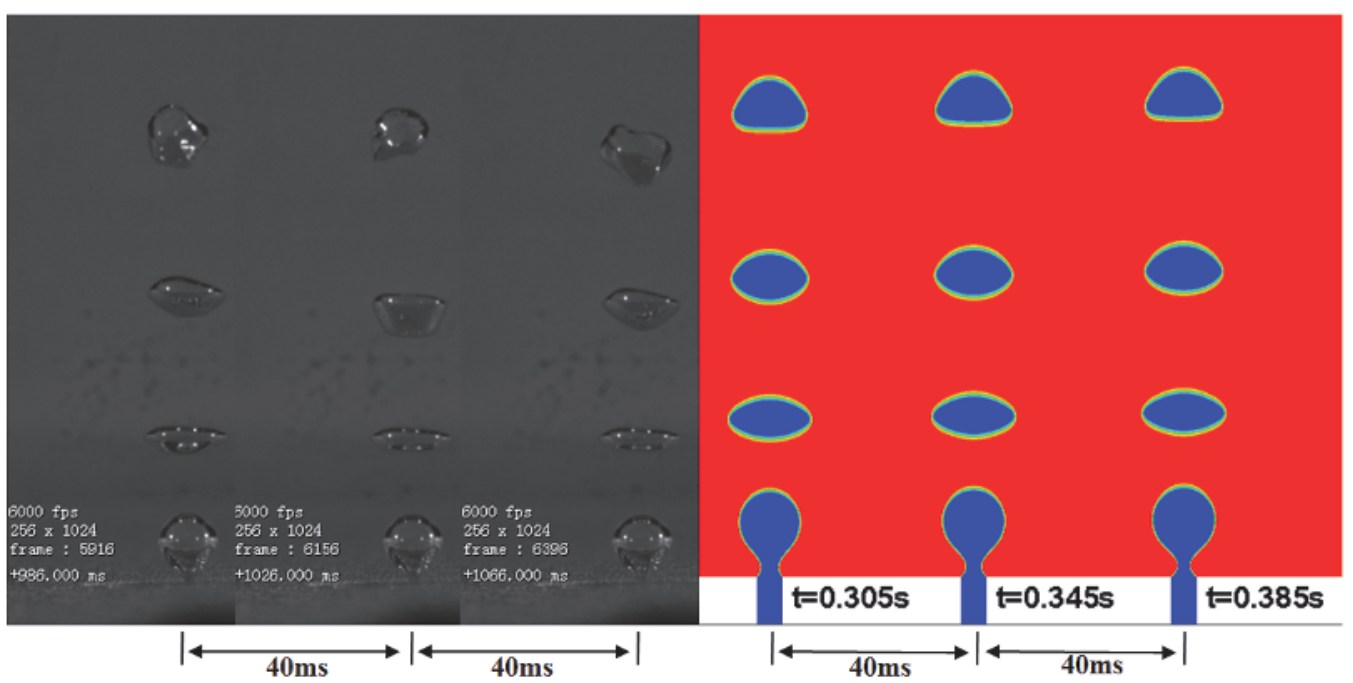

Fig. 5 Bubble evolution in simulation and experiment at gas flow rate of $100 \mathrm{ml} / \mathrm{min}$ 
detachment. In the stage I, the bubble takes a sphere shape and grows with the continuous injected gas through the channel, as shown in Fig. 7- I . When the bubble becomes large enough, the bottom part begins to shrink and a short cylindrical bubble appears, through which the whole bubble contacts with the orifice, as shown in Fig. 7- II. When it is stretched to a certain length, the cylindrical bubble will lose stability, shrinking inwards continuously until detaches from the orifice, as shown in Fig. 7-III. The detached bubble rises up in the water with time until it reaches the water surface.

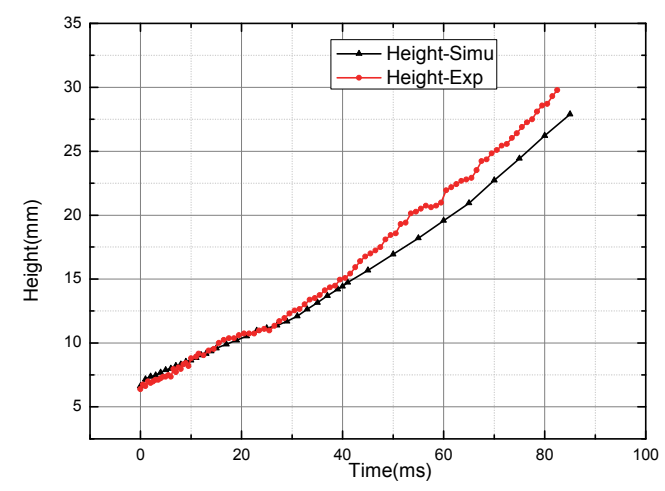

Fig. 7 Bubble rise height in experiment and simulation

The three stage will be shown in detail in the following part, especially the stage III - bubble detachment.

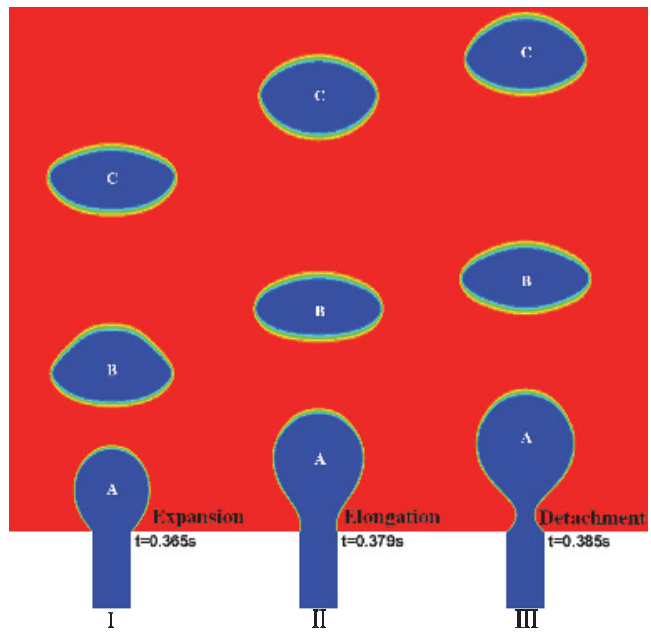

Fig. 8 Bubble formation in a single period

\subsubsection{Bubble expansion}

The bubble expansion was extensively studied. A brief description of this stage is presented here. When the gas flow rate is low, the drag force could be neglected ${ }^{[2]}$. The bubble is in equilibrium under the surface tension and the buoyancy. The expansion of the bubble is thought to be quasi-static. But when the gas flow rate is a little higher, such as the case in this paper, the bubble in the expansion stage is acted jointly by the buoyancy $F_{B}$, the surface tension $F_{\sigma}$, the drag force $F_{D}$ and the gas momentum force $F_{M}$ ${ }^{[2][3]}$, as shown in Fig. 8. At a medium gas flow rate, the interaction between bubbles seems negligible during the formation. The bubble grows in the radial direction due to the incoming gas flux and the bubble base remains attached to the orifice. The growth rate of the bubble volume is nearly equal to the gas flow rate. With the gas injection, the spherical bubble goes into the elongation stage when the downward resultant force(the surface tension $F_{\sigma}$ and the drag force $F_{D}$ ) is larger than the upward resultant force (the buoyancy $F_{B}$ and the gas momentum force $F_{M}$ ), i.e. $F_{B+} F_{M} \geq F_{\sigma}+F_{D}$.

\subsubsection{Bubble elongation}

During the elongation stage, additional gas is fed into the bubble and the bubble continues to grow in size. The bubble rises away from the orifice, but still attached to it through a neck, which is thought to be a

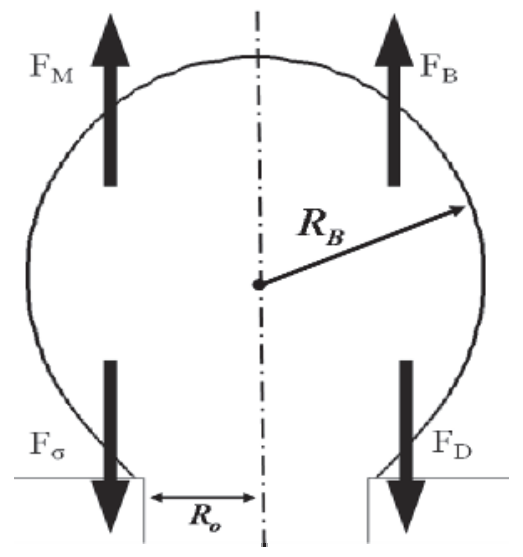

Fig. 9 The analysis of forces on bubble at medium gas low rate

stable cylindrical bubble, as shown in Fig. 7- II . Along X-coordinate axis, the forces is in balance all the time. The forces on Y-coordinate axis is shown in Fig. 9, where $P_{i n}, P_{\text {out }}$ are the pressures in and outside the bubble, and $F_{\sigma-i n}$ is the surface tension. In the elongation stage, the neck is in equilibrium under these forces and will go into the detachment stage once it loses stability.

For the bulking of the slim bar, the bar is thought to lose stability if it can not regain the original state after a small disturbance. The cylindrical bubble is assumed to get a very small disturbance and its surface tension changes, as shown in Fig. 10. The surface tension can be decomposed into $F_{\sigma-o u t}$ and $F_{\sigma-i n}$, and $F_{\sigma-o u t}$ could help the neck to recover while $F_{\sigma-i n}$ prevents it. Thus the bubble neck will 
lose stability and goes into the detachment stage if $\Delta F_{\sigma-o u t} \leq \Delta F_{\sigma-\text { in }}$.

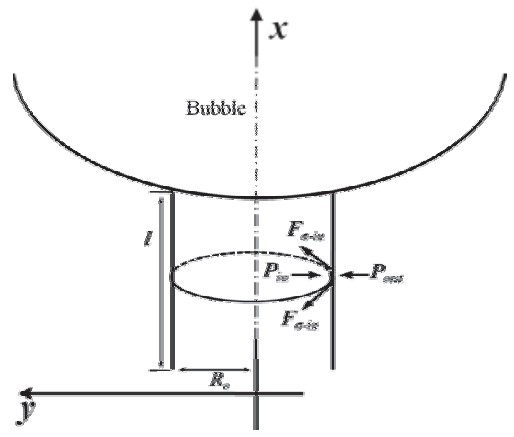

Fig. 10 The analysis of forces on the stable cylindrical

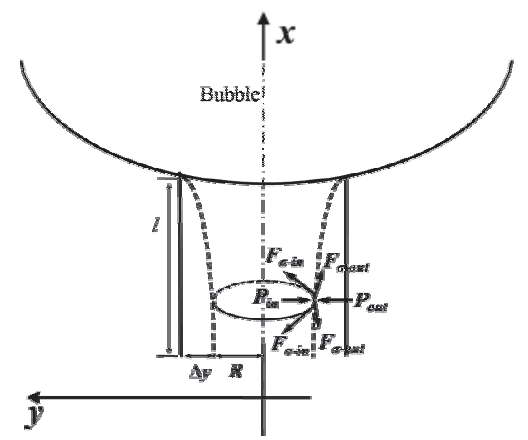

Fig. 11 The analysis of forces on the unstable cylindrical bubble

The differential pressure increases with depth, a small lateral displacement $\Delta y$ is assumed to take place at the bottom of the bubble neck, then

$$
\begin{aligned}
& \Delta F_{\sigma-\text { out }}=\frac{\sigma}{R_{\text {out }}} \\
& \Delta F_{\sigma-\text { in }}=\frac{\sigma}{R}-\frac{\sigma}{R_{o}}
\end{aligned}
$$

where $R_{o}$ is the radius of the orifice, $R$ is the radius of the bubble neck, apparently, $R=R_{o}-\Delta y . \sigma_{\text {is }}$ the surface tension coefficient. $R_{\text {out }}$ is the curvature radius corresponding to $F_{\sigma-o u t}$. The curve of the neck bubble after a small disturbance is assumed to be a part of a circle,

$$
\frac{\Delta y}{\sqrt{\Delta y^{2}+l^{2}}}=\frac{\sqrt{\Delta y^{2}+l^{2}}}{2 R_{\text {out }}}
$$

so $\quad R_{\text {out }}=\frac{l^{2}+\Delta y^{2}}{2 \Delta y} . \Delta y$ is relatively small compared with $l$ and $\Delta y^{2}$ can be neglected,
$R_{\text {out }} \approx \frac{l^{2}}{2 \Delta y}$. Combined with the condition $\Delta F_{\sigma-o u t} \leq \Delta F_{\sigma-\text { in }}$, we can have the criterion of instability:

$l \geq \sqrt{2} R_{o}$

The bubble neck will go into the detachment stage if the neck length is larger than or equal to $\sqrt{2}$ times of the orifice's radius.

The above criterion is validated by simulation. Fig. 11 and Table 1 show the critical $l_{\mathrm{c}}$ in the simulation and calculated by Eq.(26) with different gas flow rates and orifice diameters. The case 2 and the case 3 are obtained from the simulation while the case 1 is from Ref. [27].

\subsubsection{Bubble detachment}

According to Eq.(26), when the length of the bubble neck is larger than $\sqrt{2} R_{o}$, the bubble will go into the detachment stage. The cylindrical bubble neck will shrink until the entire bubble separates from the orifice, as shown in Fig. 12.

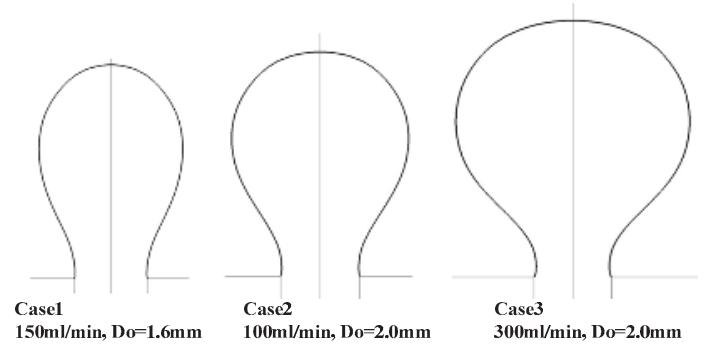

Fig. 12 Simulation validation of bubble neck's stability criterion

Table 1 Simulation validation of bubble neck's stability criterion

\begin{tabular}{lcllll}
\hline & $R_{o}$ & $\begin{array}{l}\text { Flow } \\
\text { Rate } \\
\mathrm{ml} / \mathrm{mi} \\
\mathrm{nm}\end{array}$ & $\begin{array}{l}\text { Simu- } \\
l_{c} \\
\mathrm{~mm}\end{array}$ & $\begin{array}{l}\text { Eq.(26)- } \\
l_{c} \\
\mathrm{~mm}\end{array}$ & Error \\
& & 2.5 & 1.26 & 1.13 & $-11.4 \%$ \\
Case1 $^{[27]}$ & 0.8 & 2.5 & 1.41 & $11.6 \%$ \\
Case2 & 1.0 & 100 & 1.25 & $1.0 \%$ \\
Case3 & 1.0 & 300 & 1.16 & 1.41 & $18.0 \%$ \\
\hline
\end{tabular}

According to Burton ${ }^{[2]}$, the cylindrical coordinate version of the general Rayleigh-Plesset equation can be used to describe the bubble dynamics near the necking region.

$$
\begin{aligned}
\frac{P_{o u t}-P_{\text {in }}}{\rho_{\text {ext }}}+\frac{2 \mu_{\text {ext }} \dot{R}+\sigma}{\rho_{\text {ext }} R}= & \left(\ddot{R} R+\dot{R}^{2}\right) \ln \frac{R}{r} \\
& +\frac{1}{2} \dot{R}^{2}
\end{aligned}
$$


$\mu_{\text {ext }}$ and $\rho_{\text {ext }}$ are the viscosity and the density of the exterior fluid, $\mathrm{r}$ is somewhere in the range of $r \gg R$. The rising motion of the bubble is very fast compared to the whole formation process according to Burton $^{[2]}$. Thus the effect of the viscosity could be neglected, and Eq.(27) can be written as

$$
\rho_{\text {ext }}\left[\left(\ddot{R} R+\dot{R}^{2}\right) \ln \frac{R}{r}+\frac{1}{2} \dot{R}^{2}\right]=\left[P_{\text {out }}-P_{\text {in }}\right]+\frac{\sigma}{R}
$$

When the bubble neck is in a cylindrical shape, as shown in Fig. 9, $\frac{\sigma}{R}$ stands for the surface tension $F_{\sigma-i n}$ pointing to the bubble inside, and the curvature radius in the normal direction tends to infinity. But when the cylindrical bubble neck shrinks, it turns into a saddle surface, as shown in Fig. 13 and Fig. 10, and the curvature radius in the normal direction changes. We make a correction of the surface tension by adding $F_{\sigma-o u t}$, i.e. $\frac{\sigma}{R_{\text {out }}}$, which stands for the surface tension pointing to the bubble outside.

$$
\begin{aligned}
\rho_{\text {ext }}\left[\left(\ddot{R} R+\dot{R}^{2}\right) \ln \frac{R}{r}+\frac{1}{2} \dot{R}^{2}\right] & =\left[P_{\text {out }}-P_{\text {in }}\right] \\
& +\left(\frac{\sigma}{R}-\frac{\sigma}{R_{\text {out }}}\right)
\end{aligned}
$$

This is a second order differential equation, the left side stands for the transformation of the bubble and the right side is the resultant force. With the unknown parameter of the integral limit $\mathrm{r}$ in $\ln \frac{R}{r}$, Eq.(29) can not be solved. We may give it an appropriate value to go through the simulation.

We could obtain the bubble profile and the pressure field against time by simulation, as shown inFig. 14. From the pressure field, $P_{\text {out }}$ and $P_{\text {in }}$ are known, both perpendicular to the bubble surface. From the bubble profile, the curvature of the neck can be obtained and thus the surface tension $\frac{\sigma}{R}$, and $\frac{\sigma}{R_{\text {out }}}$ is known where $\sigma$ is 0.07 for the water. As a result, the right side of Eq.(29) in the simulation can be determined.

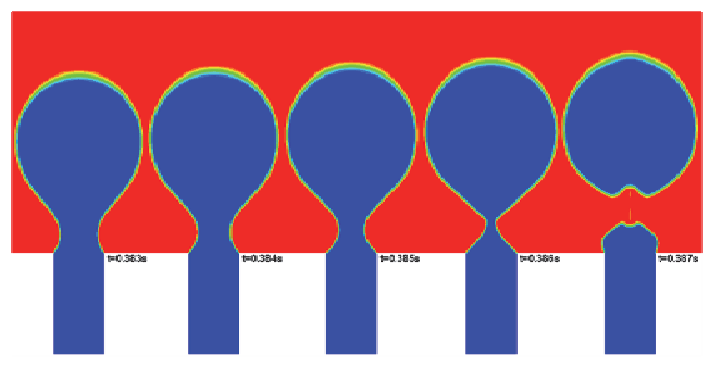

Fig. 13 Bubble detachment

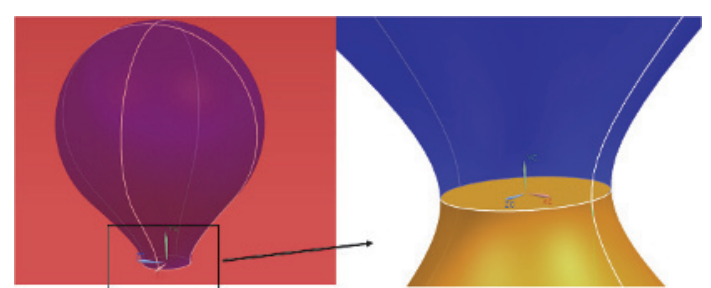

Fig. 14 Cylindrical bubble neck is part of saddle surface

On the left side of Eq.(29), the minimum radius of the neck $R_{\min }$ is the minimum value of Y (bubble profile) in Fig. 14. So $R_{\min }$ and its variation $\dot{R}_{\min }$ and $\ddot{R}_{\min }$ can be obtained. If $\mathrm{r}$ is given, the left side of Eq.(29) for $R_{\min }$ will be known. When both sides of Eq.(29) are very close, the value of $r$ is what we want. This is the idea of assuming an appropriate $r$ in this paper.

According to $\ln \frac{R}{r}$ in Eq.(29), $\mathrm{r}$ is assumed as $r=R \cdot e^{n}, n=1,1.5,2,3,4$. The comparison between the two sides of Eq.(29) at different $r$ is shown in Fig. 15 . One can see that the values of both sides are very close when $n=1.5 \sim 2$. So we think that $r$ in the cylindrical coordinate version Rayleigh-Plesset equation for the bubble pinch-off is about $R \cdot e^{1.5} \leq r \leq R \cdot e^{2}$,i.e. $4.5 R \leq r \leq 7.4 R$. Eq.(29) can be written as

$$
\begin{aligned}
\rho_{\text {ext }}\left[C \cdot\left(\ddot{R} R+\dot{R}^{2}\right)+\frac{1}{2} \dot{R}^{2}\right] & =\left[P_{\text {out }}-P_{\text {in }}\right] \\
& +\left(\frac{\sigma}{R}-\frac{\sigma}{R_{\text {out }}}\right)
\end{aligned}
$$

where $4.5 \leq C \leq 7.4$. Eq. (30) could be used to predict the dynamic behaviors of the gas bubble formation in the water. Eq.(30) still needs a further detailed experimental validation. 


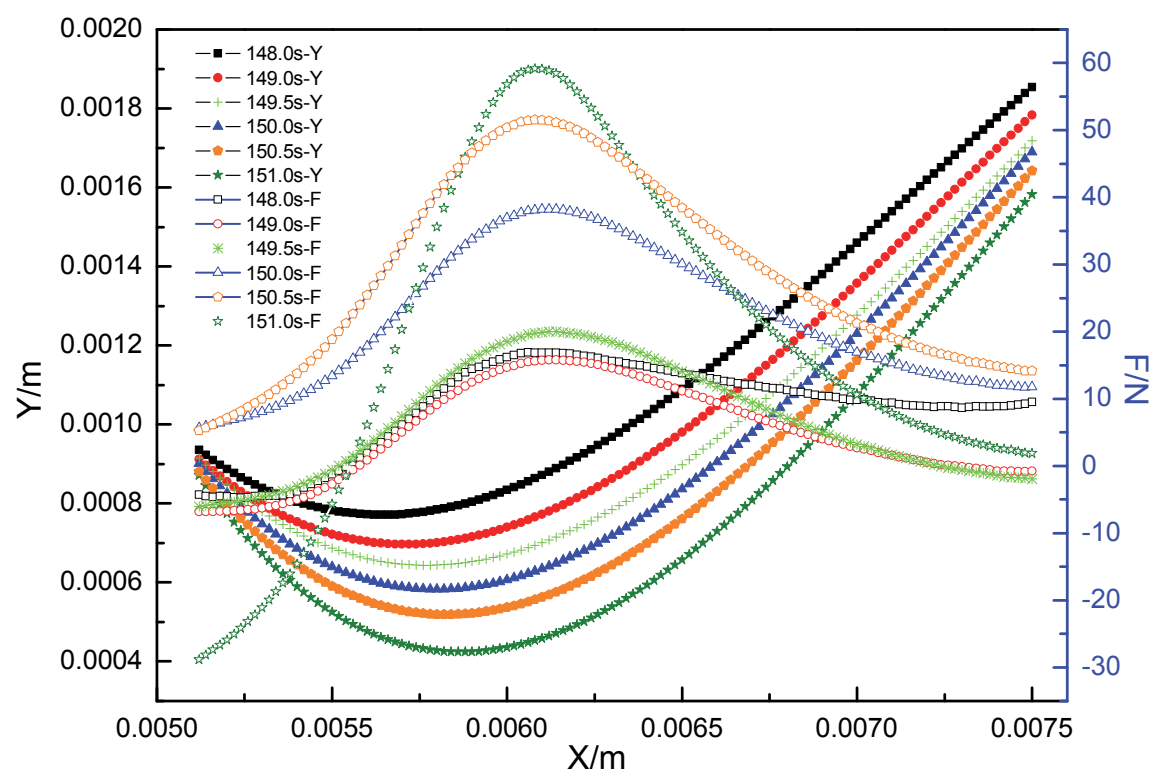

Fig. 15 Profile of bubble neck and responding resultant force against time

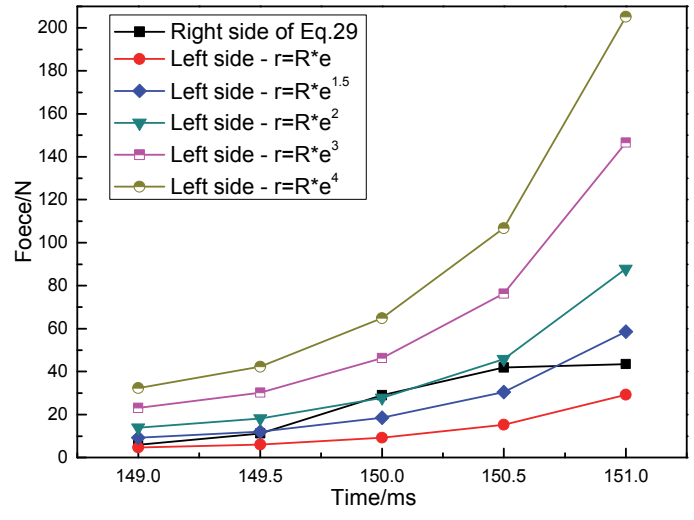

Fig. 16 Comparison between the two sides of Eq.(29) at different $r$

\section{Conclusions}

Experiment and simulation of the bubble formation on a submerged orifice at a constant gas flow rate are carried out in this paper. The compressible large eddy simulation combined with the volume of fluid (VOF) is adopted in the simulation and the results are validated by experiment. The bubble formation experiences three stages in this paper, the expansion stage, the elongation stage and the detachment stage.

In the expansion stage, the bubble grows in the radial direction due to the incoming gas flux and the bubble base is attached to the orifice. The growth rate of the bubble volume is nearly equal to the gas flow rate. With the gas injection, the spherical bubble will go into the elongation stage. In this stage, the bubble rises away from the orifice, but is still attached to it through a neck, which is thought to be a stable cylindrical bubble.
The criterion of the bubble neck stability in the elongation stage is obtained. Its surface tension will change when the bubble neck has a small displacement. If $\Delta F_{\sigma-\text { out }} \leq \Delta F_{\sigma-\text { in }}$, the bubble will lose stability. At last, the instability criterion is obtained that the bubble will go into the detachment stage once the bubble neck's length is larger than or equal to $\sqrt{2}$ times of the orifice radius, i.e. $l \geq \sqrt{2} R_{o}$. This criterion is also validated by simulation in this paper.

The dynamics behaviors of the bubble neck interface during the detachment are analyzed. The cylindrical coordinate Rayleigh-Plesset equation can be used to describe the detachment stage. The forces and the deformation of the bubble neck in the Rayleigh-Plesset equation can be obtained from simulation. Therefore the unknown parameter of the integral limit $\mathrm{r}$ in the equation can be estimated. $\mathrm{r}$ is given as $R \cdot e^{1.5} \leq r \leq R \cdot e^{2}$ according to the simulation results in this paper.

\section{References}

[1] Zhang L., Shoji M. Aperiodic bubble formation from a submerged orifice [J]. Chemical Engineering Science, 56(18): 5371-5381.

[2] Legendre D., Zenit R., Velez-Cordero J. R. On the deformation of gas bubbles in liquids [J]. Physics of Fluids, 2012, 24: 043303.

[3] Legendre D. On the relation between the drag and the vorticity produced on a clean bubble [J]. Physics of Fluids, 2007, 19: 018102.

[4] Gerlach D., Biswas G., Durst F. et al. Quasi-static bubble formation on submerged orifices [J]. International Journal of Heat and Mass Transfer, 2005, 48(2): 425-438.

[5] Buwa V. V., Gerlach D., Durst F. et al. Numerical simulations of bubble formation on submerged orifices: 
Period-1 and period-2 bubbling regimes [J]. Chemical Engineering Science, 2007, 62(24): 7119-7132.

[6] Gerlach D., Alleborn N., Buwa V. et al. Numerical simulation of periodic bubble formation at a submerged orifice with constant gas flow rate [J]. Chemical Engineering Science, 2007, 62(7): 2109-2125.

[7] Kulkarni A. A., Joshi J. B. Bubble formation and bubble rise velocity in gas-liquid systems: A review [J]. Industrial \& Engineering Chemistry Research, 2005, 44(16): 5873-5931.

[8] Jamialahmadi M., Zehtaban M., Müller-Steinhagen H. et al. Study of bubble formation under constant flow conditions [J]. Chemical Engineering Research \& Design, 2001, 79: 523-532.

[9] Di Bari S., Robinson A. J. Experimental study of gas injected bubble growth from submerged orifices [J]. Experimental Thermal and Fluid Science, 2013, 44(1): 124-137.

[10] Hysing S., Turek S., Kuzmin D. et al. Quantitative benchmark computations of two-dimensional bubble dynamics [J]. International Journal for Numerical Methods in Engineering, 2009, 60(11): 1259-1288.

[11] Zhu X., Xie J., Liao Q. et al. Dynamic bubbling behaviors on a micro-orifice submerged in stagnant liquid [J]. International Journal of Heat and Mass Transfer, 2014, 68: 324-331.

[12] Xie J., Zhu X., Liao Q. et al. Dynamics of bubble formation and detachment from an immersed micro-orifice on a plate [J]. International Journal of Heat and Mass Transfer, 2012, 55(11-12): 3205-3213.

[13] Martín M., García J. M., Montes F. J. et al. On the effect of the orifice configuration on the coalescence of growing bubbles [J].Chemical Engineering and Processing, 2008, 47(9): 1799-1809.

[14] Zhang J., Yu Y., Qu C. et al. Experimental study and numerical simulation of periodic bubble formation at submerged micron-sized nozzles with constant gas flow rate [J]. Chemical Engineering Science, 2017, 168: 1-10.

[15] Vokurka K. Significant intervals of energy transforms in bubbles freely oscillating in liquids [J]. Journal of Hydrodynamics, 2017, 29(2): 217-225.
[16] Sarhan A. R., Naser J., Brooks G. CFD modeling of bubble column: Influence of physico-chemical properties of the gas/liquid phases properties on bubble formation [J] Separation and Purification Technology, 2018, 201: 130-138.

[17 Burton J., Waldrep R., Taborek P. Scaling and instabilities in bubble pinch-off [J]. Physical Review Letters, 2005, 94: 184502.

[18] Gordillo J., Sevilla A., Rodriguez-Rodriguez J. et al. Axisymmetric bubble pinch-off at high Reynolds numbers [J]. Physical Review Letters, 2005, 95: 194501.

[19] Leppinen D., Lister J. R. Capillary pinch-off in inviscid fluids [J]. Physics of Fluids, 2003, 15: 568.

[20] Thoroddsen S., Etoh T., Takehara K. Experiments on bubble pinch-off [J]. Physics of Fluids, 2007, 19: 042101.

[21] Gordillo J. Axisymmetric bubble collapse in a quiescent liquid pool. I. Theory and numerical simulations [J]. Physics of Fluids, 2008, 20: 112103.

[22] Bolaños-Jiménez R., Sevilla A., Martinez-Bazan C. et al. Axisymmetric bubble collapse in a quiescent liquid pool. II. Experimental study [J]. Physics of Fluids, 2008, 20: 112104.

[23] Quan S., Hua J. Numerical studies of bubble necking in viscous liquids [J]. Physical Review E, 2008, 066303.

[24] Garnier E., Adams N., Sagaut P. Large eddy simulation for compressible flows [M].Springer Science \& Business Media, 2009.

[25] Lin W. Large-eddy simulation of premixed turbulent combustion using flame surface density approach [D]. School of Graduate Studies - Theses, 2011.

[26] Chen C., Fan L. S. Discrete simulation of gas-liquid bubble columns and gas - liquid - solid fluidized beds [J]. AIChE Journal, 2004, 50(2): 288-301.

[27] Albadawi A., Donoghue D., Robinson A. et al. On the analysis of bubble growth and detachment at low capillary and bond numbers using volume of fluid and level set methods [J]. Chemical Engineering Science, 2013, 90(Complete): 77-91. 